\title{
High Power Linearly Polarized Raman Fiber Laser With Stable Temporal Output
}

\author{
Jiaxin SONG*, Hanshuo WU, Jun YE, Jiangming XU, \\ Hanwei ZHANG, and Pu ZHOU
}

\author{
College of Advanced Interdisciplinary Studies, National University of Defense Technology, Changsha 410073, China \\ ${ }^{*}$ Corresponding author: Jiaxin SONGＩE-mail: songjiaxin1994@163.com
}

\begin{abstract}
We demonstrate a high power linearly polarized Raman fiber laser (RFL) pumped by an amplified spontaneous emission (ASE) source. Temporal-stable operation of RFL could be ensured owing to the employment of ASE, which mitigates the inherent intensity noise compared with the classic scheme adopting laser oscillator as pump source. In this experiment, the RFL has up to $119.5 \mathrm{~W}$ output power, with central wavelength of $1129.2 \mathrm{~nm}$, and full width at half maximum (FWHM) linewidth of about $4.18 \mathrm{~nm}$. The polarization extinction ratio (PER) of the Raman laser is about $23 \mathrm{~dB}$. Moreover, this laser has excellent long-term and short-term stabilities in terms of the output power and time domain.
\end{abstract}

Keywords: Raman fiber laser; amplified spontaneous emission; linearly polarized

Citation: Jiaxin SONG, Hanshuo WU, Jun YE, Jiangming XU, Hanwei ZHANG, and Pu ZHOU, "High Power Linearly Polarized Raman Fiber Laser With Stable Temporal Output," Photonic Sensors, 2019, 9(1): 43-48.

\section{Introduction}

Raman fiber lasers (RFLs) have attracted much attention in the past few decades owing to their advantages of wavelength flexibility, compact structure, high power handling capacity, etc. [1], and have already found a wide range of applications in optical pumping, frequency conversion, optical communications, fiber sensing, biology, and supercontinuum generation [2-8]. Up to date, the investigation into RFLs has covered many areas, including power scaling $[9,10]$, linewidth narrowing [11, 12], temporal characteristics [13, 14], polarization state [15], and working wavelength expansion [16, 17], among which the temporal stability is an important aspect that affects the performance of RFLs.
According to the temporal state, RFLs can be roughly classified into continuous-wave $(\mathrm{CW})$ and pulsed ones. However, as far as we know, it is not easy to obtain a true CW RFL, since the pump source (mostly rare-earth doped fiber laser) of RFL usually has self-pulsing under certain pumping and cavity conditions [18-20] that will transfer to the RFL [21, 22], thus resulting in a "pretended" CW RFL. This would degrade the performance of laser systems that employ RFLs as pump or seed sources. Therefore, it is of great significance to study stable CW Raman lasers. Since the temporal property can be transferred from the pump source to Raman lasing, thus adopting temporal stable pump source can hopefully contribute to achieve RFLs with stable $\mathrm{CW}$ output. Among all the available pump sources, amplified spontaneous emission source (ASE, also

Received: 25 April 2018 / Revised: 19 June 2018

(C) The Author(s) 2018. This article is published with open access at Springerlink.com

DOI: $10.1007 / \mathrm{s} 13320-018-0506-9$

Article type: Regular 
known as superfluorescent fiber source) has been proven to have high temporal stability [23-26], and can serve as an ideal pump source. Owing to the broad linewidth and stable temporal property, ASE source has also found potential applications such as rotation sensing (especially fiber gyroscopes) [27-29]. Although an ASE source in $1.5 \mu \mathrm{m}$ has been used to pump Raman fiber laser to verify its capability of suppressing stimulated Brillouin scattering and increasing the pumping efficiency [30], the temporal stability of CW operation has not yet been investigated. Therefore, it is interesting to verify the feasibility of realizing Raman lasing with stable CW output by adopting an ASE pump source. Moreover, high output power and linearly polarized operation of the proposed laser are also investigated because some applications, e.g. nonlinear frequency conversion, require both high power and linearly polarized laser to meet the threshold and improve overall efficiency.

In this paper, we demonstrate a high power linearly polarized Raman fiber laser pumped by a powerful ASE source. The maximum output power achieved is $119 \mathrm{~W}$ with a polarization extinction ratio (PER) around $23 \mathrm{~dB}$. More importantly, this laser possesses good stability, with standard deviation (STD) of $\sim 0.35 \%$ for the time domain dynamics and STD of $0.029 \%$ for the fluctuation of total output power. To the best of our knowledge, this is the first demonstration of a hundred-Watt level Raman fiber laser with relatively stable temporal output enabled by the ASE pump source.

\section{Experimental setup}

As shown in Fig. 1, the ASE pump source consists of a homemade broadband ASE seed source [31] and two-stage polarization-maintaining (PM) power amplifiers, the central wavelength of which is about $1074.8 \mathrm{~nm}$, with full width at half maximum (FWHM) linewidth $\sim 10.3 \mathrm{~nm}$ at the maximum pump power. A PM isolator (ISO) is adopted to extract linearly polarized ASE source. The output end of the
ASE source is spliced with the $1070 \mathrm{~nm}$ port of a $1070 / 1120 \mathrm{~nm}$ wavelength division multiplexer (WDM). The $1120 \mathrm{~nm}$ port of the WDM is connected to a fiber loop mirror (FLM), which is used to serve as a wideband high reflective cavity mirror. The FLM is fabricated by splicing two adjacent ports of a 50/50 coupler together, whose central working wavelength is $1120 \mathrm{~nm}$ with working bandwidth of $\sim 20 \mathrm{~nm}$. The common port of the WDM is spliced with a piece of 59-m long passive fiber $(10 / 125 \mu \mathrm{m})$, which is used to provide Raman gain. The output end of this piece of passive fiber is cleaved with a flat angle to provide feedback, and the reflectivity is around $4 \%$.

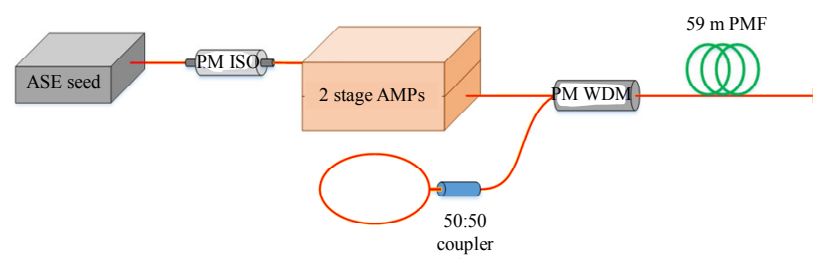

Fig. 1 Experimental setup of the Raman laser (ASE: amplified spontaneous emission, PM: polarization maintaining, ISO: isolator, AMP: amplifier, WDM: wavelength division multiplexer, PMF: polarization maintaining fiber).

\section{Results}

The power evolution of the total output, residual pump, 1st order Stokes wave, and 2nd order Stokes light are shown in Fig. 2(a), the central wavelengths of which are $1074.8 \mathrm{~nm}, 1129.2 \mathrm{~nm}$, and $1192.2 \mathrm{~nm}$, respectively. The frequency shift between the pump source and the 1st order Stokes light is about 13.4 $\mathrm{THz}$, while the frequency shift between the 1st and 2 nd order Stokes lights is about $14.0 \mathrm{THz}$. The threshold power of the 1st order Stokes light is about $60 \mathrm{~W}$. The maximum output power of $1129.2 \mathrm{~nm}$ Raman laser is $119.5 \mathrm{~W}$ under the pump power of $153.10 \mathrm{~W}$, which corresponds to the conversion efficiency of $78.05 \%$. Figure 2(b) depicts the spectra evolution of the Raman fiber laser. As the pump power increases, the 1st order Stokes light and the 2nd order Stokes light start to emerge successively. At the maximum pump power, the 1st order Stokes wave occupies $92.1 \%$ of the whole spectrum, while 
the $2 \mathrm{nd}$ order Stokes wave at $1192.18 \mathrm{~nm}$ merely accounts for $2.6 \%$, which coincides with what is shown in Fig. 2(a).

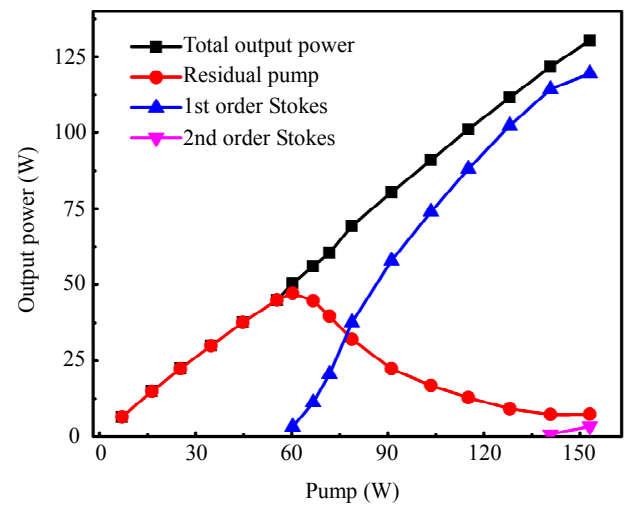

(a)

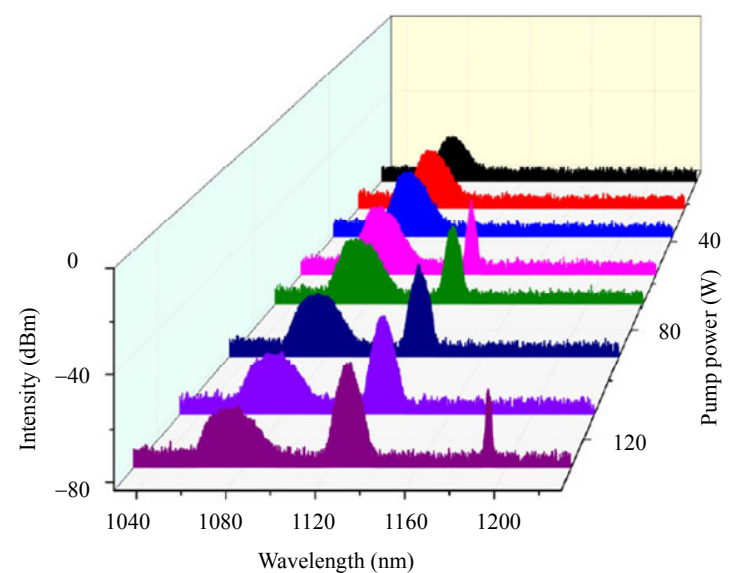

(b)

Fig. 2 Output properties: (a) the output power evolution of the Raman fiber laser and (b) the spectra evolution of the Raman fiber laser.

The linewidth evolutions of the ASE pump source, residual pump light, and the 1st and 2nd Stokes lights as a function of the pump power (i.e., the output power of the ASE source) are compared, as shown in Fig. 3. The FWHM linewidth of the ASE pump source are generally stable, which is around $8 \mathrm{~nm}$, and rises to $10.38 \mathrm{~nm}$ at the maximum pump power. While the linewidth of the 1 st order Stokes light experiences a steady growth, which increases from $0.673 \mathrm{~nm}$ to $4.18 \mathrm{~nm}$. The linewidth of the residual pump light shows a distinct different variation style. When the pump power is lower than the threshold of the 1st order Stokes light, the linewidth of the residual pump light is about $8 \mathrm{~nm}$, which is close to that of the ASE pump source.
However, the linewidth increases sharply to about $14 \mathrm{~nm}$ when the pump power is higher than the threshold of the 1st order Stokes light.

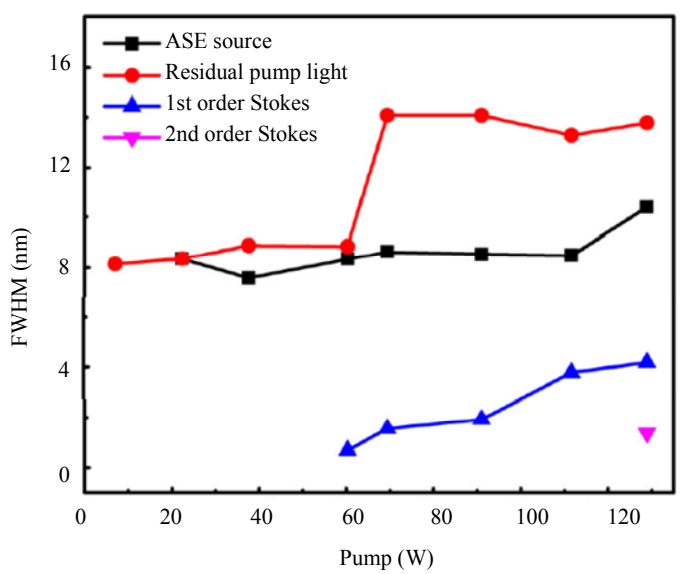

Fig. 3 Linewidth variation of the ASE source, residual pump light, and 1 st and 2 nd order Stokes lights as a function of the ASE pump power.

The PER of the ASE pump source and the output Raman laser are measured as well. The output laser goes through a collimator to guarantee the collimation property of the laser beam. Then, the first order Stokes wave is extracted from the output laser by a 1070/1120 nm dichroic mirror. A half-wave plate, the central wavelength of which is $1120 \mathrm{~nm}$, is placed after the dichroic mirror to adjust the polarization direction of the propagating wave. A polarization beam splitter working around $1120 \mathrm{~nm}$ is adopted to split the light into two perpendicular directions, and the output power of each polarization direction is then recorded by two power meters. The $P E R$ of the output laser can be calculated according to the formula as

$$
P E R=10 \lg \frac{a}{b}
$$

where $a$ and $b$ are the values of minor and major axes of the polarization ellipse, which correspond to the powers measured by the power meters. The PER of the pump source can be obtained to be about $14 \mathrm{~dB}$ by measuring the $P E R$ of the residual pump when the pump power is less than the threshold power of $\sim 60 \mathrm{~W}$, as shown in Fig. 4 . As the pump power increases to a value higher than $60 \mathrm{~W}$, the PER evolution of the 1st order Stokes 
wave is measured to be around $23 \mathrm{~dB}$, and there is no apparent degradation.

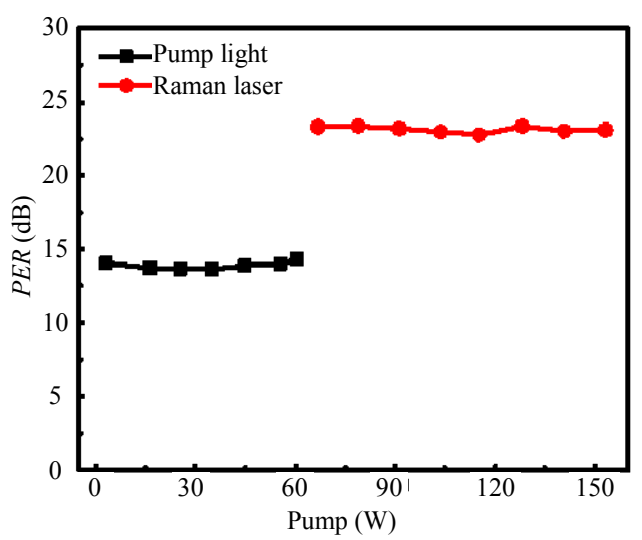

Fig. 4 PER of the pump light and the Raman laser.

The stability of output laser is also recorded and analyzed in a relatively long period and a short one, respectively. On a long-term scale, the variety of the output power is tested by the power meter at the full pump power. The maximum total output power, i.e. the residual pump power plus Stokes light power, reaches $130.33 \mathrm{~W}$, and the output power is recorded every 4 seconds in a total time duration of 5 minutes, as shown in Fig. 5. The STD of the normalized output power is only $0.029 \%$, showing the ultra-stable property of this laser.

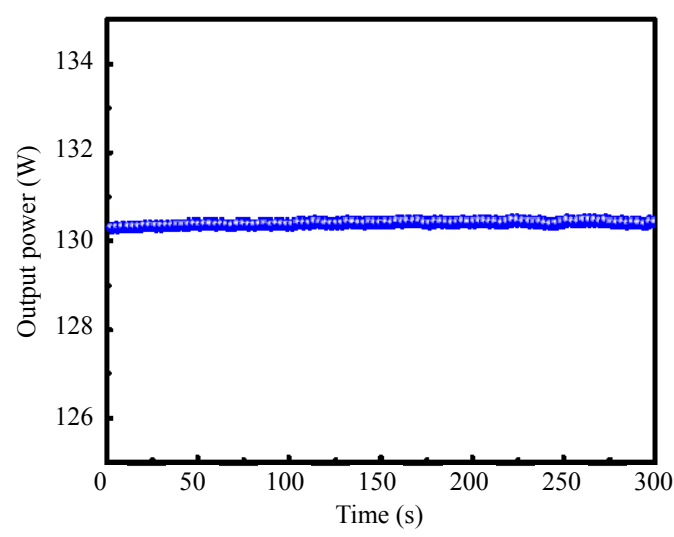

Fig. 5 Output power stability in a time scale of $300 \mathrm{~s}$.

On a short-term scale, Fig. 6 describes the normalized temporal dynamic of the laser output in the timescale of 1 millisecond. The STD of the temporal sequence is about $0.35 \%$, which indicates the temporal characteristics are very stable.

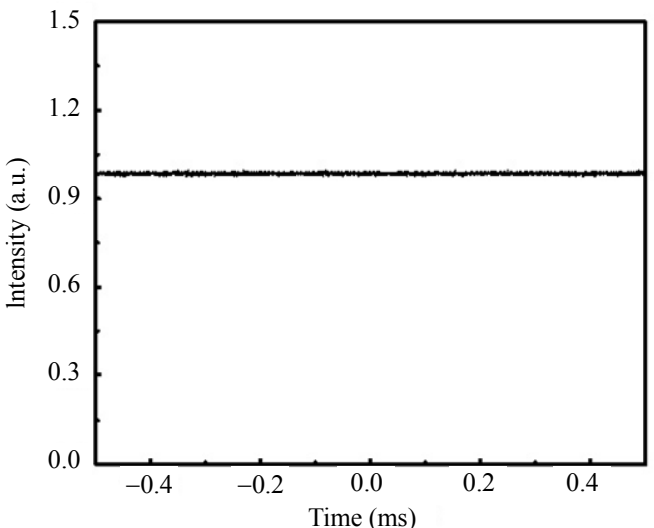

Fig. 6 Normalized temporal dynamics of the laser at the maximum output power

\section{Discussion}

In this paper, a hundred-watt level linearly polarized Raman fiber laser is obtained with an ASE pump source. When the 1st order Stokes light occurs, the linewidth of the residual pump light increases to about $14 \mathrm{~nm}$. Such rapid linewidth broadening of the residual pump light can be explained by the following reason: the wavelength near the spectrum center of the ASE source has higher power and will reach the power level for stimulated Raman lasing first. In this case, the power at the central wavelengths of the pump source would transfer to the 1st order Stokes light and the intensity of these wavelengths decreases to a power level close to the wavelengths without Raman lasing, thus the linewidth of the residual pump light shows a tendency of broadening. The output light has very high PER (about $23 \mathrm{~dB}$ ), which could possibly be attributed to the Raman purification effect. Owing to the high temporal stability of the ASE source, quite stable CW Raman laser is obtained both on the long-term and short-term scales.

\section{Conclusions}

In summary, we have demonstrated an ASE pumped, temporal stable, and high power linearly polarized Raman fiber laser. The proposed Raman fiber laser can deliver up to $119.5 \mathrm{~W}$ output power at $1129.2 \mathrm{~nm}$, corresponding to the conversion efficiency of $\sim 78.05 \%$. The long-term and 
short-term stability of the intensities of the output laser are tested in 5 minutes and 1 microseconds, respectively, and the results indicate quite good stability. The STD of the normalized temporal dynamics is only $0.35 \%$. and that of the normalized output power is only $0.029 \%$. The PER of the Raman laser reaches about $23 \mathrm{~dB}$, although the PER of the ASE source is relatively low (about $14 \mathrm{~dB}$ ). To the best of our knowledge, this is the first time that a hundred-Watt level linearly polarized Raman fiber laser has been demonstrated enabled by an ASE pump source. Further power scaling can be expected by optimizing the length of the passive fiber and changing the reflectivity of the cavity mirrors. Moreover, this temporal stable CW Raman laser could provide a useful tool for many other applications.

Open Access This article is distributed under the terms of the Creative Commons Attribution 4.0 International License (http://creativecommons.org/licenses/by/4.0/), which permits unrestricted use, distribution, and reproduction in any medium, provided you give appropriate credit to the original author(s) and the source, provide a link to the Creative Commons license, and indicate if changes were made.

\section{References}

[1] V. R. Supradeepa, Y. Feng, and J. W. Nicholson, "Raman fiber lasers," Journal of Optics, 2017, 19(2): 023001-1-023001-26.

[2] A. E. El-Taher, J. D. Ania-Castañón, V. Karalekas, and P. Harper, "High efficiency supercontinuum generation using ultra-long Raman fiber cavities," Optics Express, 2009, 17(20): 17909-17915.

[3] L. R. Taylor, Y. Feng, and D. B. Calia, "50W CW visible laser source at $589 \mathrm{~nm}$ obtained via frequency doubling of three coherently combined narrow-band Raman fibre amplifiers," Optics Express, 2010, 18(8): 8540-8555.

[4] X. Wang, P. Zhou, Y. Miao, H. W. Zhang, H. Xiao, X. L. Wang, et al., "Raman fiber laser-pumped high-power, efficient Ho-doped fiber laser," Journal of the Optical Society of America B, 2014, 31(10): 2476-2479.

[5] H. W. Baac, N. Uribe-Patarroyo, and B. E. Bouma, "High-energy pulsed Raman fiber laser for biological tissue coagulation," Optics Express, 2014,
22(6): 7113-7123.

[6] H. W. Zhang, P. Zhou, X. Wang, X. Y. Du, H. Xiao, and $\mathrm{X}$. J. $\mathrm{Xu}$, "Hundred-watt-level high power random distributed feedback Raman fiber laser at $1150 \mathrm{~nm}$ and its application in mid-infrared laser generation," Optics Express, 2015, 23(13): 17138-17144.

[7] Z. N. Wang, Y. J. Rao, H. Wu, P. Y. Li, Y. Jiang, X. H. Jia, et al., "Long-distance fiber-optic point-sensing systems based on random fiber lasers," Optics Express, 2012, 20(16): 17695-17700.

[8] X. H. Jia, Y. J. Rao, Z. N. Wang, W. L. Zhang, C. X. Yuan, X. D. Yan, et al., "Distributed Raman amplification using ultra-long fiber laser with a ring cavity: characteristics and sensing application," Optics Express, 2013, 21(18): 21208-21217.

[9] S. A. Skubchenko, M. Y. Vyatkin, and D. V. Gapontsev, "High-power CW linearly polarized all-fiber Raman laser," IEEE Photonics Technology Letters, 2004, 16(4): 1014-1016.

[10] H. W. Zhang, P. Zhou, H. Xiao, and X. J. Xu, "Efficient Raman fiber laser based on random Rayleigh distributed feedback with record high power," Laser Physics Letters, 2014, 11(7): 075104-1-075104-4.

[11] Y. Feng, L. Taylor, and D. B. Calia, "Multiwatts narrow linewidth fiber Raman amplifiers," Optics Express, 2008, 16(15): 10927-10932.

[12] D. Georgiev, V. P. Gapontsev, A. G. Dronov, M. Y. Vyatkin, A. B. Rulkov, S. V. Popov, et al., "Watts-level frequency doubling of a narrow line linearly polarized Raman fiber laser to $589 \mathrm{~nm}$," Optics Express, 2005, 13(18): 6772-6776.

[13] A. E. Bednyakova, O. A. Gorbunov, M. O. Politko, S. I. Kablukov, S. V. Smirnov, D. V. Churkin, et al., "Generation dynamics of the narrowband Yb-doped fiber laser," Optics Express, 2013, 21(7): 8177-8182.

[14] J. Schroder and S. Coen, "Observation of high-contrast, fast intensity noise of a continuous wave Raman fiber laser," Optics Express, 2009, 17(19): 16444-16449.

[15] J. H. Wang, L. Zhang, J. Zhou, L. Si, J. B. Chen, and Y. Feng, "High power linearly polarized Raman fiber laser at $1120 \mathrm{~nm}$," Chinese Optics Letters, 2012, 10(2): 021406-1-021406-3.

[16] L. Zhang, H. W. Jiang, X. Z. Yang, W. W. Pan, S. Z. Cui, and Y. Feng, "Nearly-octave wavelength tuning of a continuous wave fiber laser," Scientific Reports, 2017, 7: 42611-1-42611-4.

[17] Z. N. Wang, H. Wu, M. Q. Fan, Y. J. Rao, X. H. Jia, and W. L. Zhang, "Third-order random lasing via Raman gain and Rayleigh feedback within a 
half-open cavity," Optics Express, 2013, 21(17): 20090-1-20090-6.

[18] W. H. Loh and J. P. de Sandro, "Suppression of self-pulsing behavior in erbium-doped fiber lasers with resonant pumping: experimental results," Optics Letters, 1996, 21(18): 1475-1477.

[19] J. L. Li, K. I. Ueda, M. Musha, and A. Shirakawa, "Residual pump light as a probe of self-pulsing instability in an ytterbium-doped fiber laser," Optics Letters, 2006, 31(10): 1450-1452.

[20] Y. L. Tang and J. Q. Xu, "Effects of excited-state absorption on self-pulsing in Tm3+-doped fiber lasers," Journal of the Optical Society of America B, 2010, 27(2): 179-186.

[21] C. R. S. Fludger, V. Handerek, and R. J. Mears, "Pump to signal RIN transfer in Raman fiber amplifiers," Journal of Lightwave Technology, 2001, 19(8): 1140-1148.

[22] M. Krause, S. Cierullies, H. Renner, and E. Brinkmeyer, "Pump-to-Stokes RIN transfer in Raman fiber lasers and its impact on the performance of co-pumped Raman amplifiers," Optics Communications, 2006, 260(2): 656-661.

[23] J. M. Xu, L. J. Huang, J. Y. Leng, H. Xiao, S. F. Guo, P. Zhou, et al., "1.01 kW superfluorescent source in all-fiberized MOPA configuration," Optics Express, 2015, 23(5): 5485-5490.

[24] P. F. Ma, L. Huang, X. L. Wang, P. Zhou, and Z. J. Liu, "High power broadband all fiber superfluorescent source with linear polarization and near diffraction-limited beam quality," Optics Express, 2016, 24(2): 1082-1088.

[25] J. M. Xu, Z. K. Lou, J. Ye, J. Wu, J. Y. Leng, H. $\mathrm{Xiao}$, et al., "Incoherently pumped high-power linearly-polarized single-mode random fiber laser: experimental investigations and theoretical prospects," Optics Express, 2017, 25(5): 5609-5617.

[26] P. Wang and W. A. Clarkson, "High-power, single-mode, linearly polarized, ytterbium-doped fiber superfluorescent source," Optics Letters, 2007, 32(17): 2605-2607.

[27] K. Iwatsuki, "Excess noise reduction in fiber gyroscope using broader spectrum linewidth Er-doped superfluorescent fiber laser," IEEE Photonics Technology Letters, 1991, 3(3): 281-283.

[28] J. Nayaks, "Fiber-optic gyroscopes: from design to production [Invited]," Applied Optics, 2011, 50(25): E152-E161.

[29] W. Burns, C. L. Chen, and R. Moeller, "Fiber-optic gyroscopes with broad-band sources," Journal of Lightwave Technology, 1983, 1(1): 98-105.

[30] B. Levit, A. Bekker, V. Smulakovsky, and B. Fischer, "Amplified-spontaneous-emission pumped raman fiber laser," in Proceeding of Conference on Lasers \& Electro-optics, Baltimore, United States, 2008, pp. 563-565.

[31] J. M. Xu, W. Liu, J. Y. Leng, H. Xiao, S. F. Guo, P. Zhou, et al., "Power scaling of narrowband high-power all-fiber superfluorescent fiber source to 1.87 kW," Optics Letters, 2015, 40(13): 2973-2976. 